\title{
Stochastic stabilisation and power control for nonlinear feedback loops communicating over lossy wireless networks
}

\author{
Alejandro I. Maass, Dragan Nešić, Vineeth S. Varma, Romain Postoyan, and Samson Lasaulce
}

\begin{abstract}
We study emulation-based stabilisation of nonlinear networked control systems communicating over multiple wireless channels subject to packet loss. Specifically, we establish sufficient conditions on the rate of transmission that guarantee $\mathcal{L}_{p}$ stability-in-expectation of the overall closed-loop system. These conditions depend on the cumulative dropout probability of the network nodes for static protocols. We use the obtained stability results to study power control, where we show there are interesting trade-offs between the transmission rate, transmit power, and stability. Lastly, numerical examples are presented to illustrate our results.
\end{abstract}

\section{INTRODUCTION}

Wireless networks are being increasingly used in control systems given the recent advances in wireless sensing, highperformance computing, and cloud technology, see e.g. [1]. Understanding the interplay between the network and control loop components is essential. In this context, packet loss has been one of the most studied communication constraints in the literature regarding controller design. Various works such as [2]-[5] have studied packet dropouts for linear discretetime systems and single communication channels, i.e. one pair of transmitter and receiver. Since wireless networked control systems (WNCSs) may be composed of multiple wireless channels with different properties and thus statistics, single channel results usually have a limited applicability in practice. Multiple channels with different probabilities of successful reception per channel have been considered in e.g. [6]-[9] also for linear systems. General nonlinear WNCSs subject to stochastic packet dropouts are studied in [10]-[12]. The work [10] models the WNCS with stochastic impulsive systems and provide conditions for mean-square stability under Bernoulli distributed packet loss. A predictive control strategy is developed in [11] to stabilise discrete-time nonlinear plants under Markovian packet dropouts. Lastly, [12] provides conditions for the $\mathcal{L}_{p}$ stability-in-expectation of the WNCS under Bernoulli dropouts and different types of scheduling. In [10]-[12], one wireless channel for the communication between controller and plant is considered.

This work was supported in part by the Australian Research Council through the Discovery Project under Grant DP170104099, and in part by the Agence Nationale de la Recherche via grant HANDY, number ANR-18CE40-0010, and the France-Australia collaboration project IRP-ARS CNRS.

A.I. Maass and D. Nešić are with the Department of Electrical and Electronic Engineering, The University of Melbourne, Parkville, 3010, Victoria, Australia (e-mail: alejandro.maass@unimelb.edu.au; dnesic@unimelb.edu.au).

V.S. Varma, R. Postoyan, and S. Lasaulce are with the Universite de Lorraine, CNRS, CRAN, F-54000 Nancy, France (email: vineeth.satheeskumar-varma@univ-lorraine.fr; romain.postoyan@univlorraine.fr; samson.lasaulce@ centralesupelec.fr).
An important aspect of wireless channels is that the dropout effect can be compensated by adjusting the transmission power leading to an improved probability of successful reception. However, increasing the power of a transmitter increases interference between channels that communicate at the same time. Power control, as a solution to interference management, has been well studied in the wireless community in e.g. [13]-[15] without the presence of a closed-loop setting. In the control community, power control has been studied for estimation in e.g. [16]-[18], and for controller design in e.g. [19], [20]. In addition to power control, designing channel access policies may help to mitigate interference as seen in [21].

In this paper, we study controller design and power control for general nonlinear plants in which all communications between plant and controller are done through multiple wireless channels subject to packet loss. We use the emulation approach to design the controller, see e.g. [22], [23]. We assume each wireless channel has its own successful reception probability which can be modified by adapting the transmitting power if required. Under almost surely exponentially stable protocols, we provide sufficient conditions on the rate of transmission that guarantee $\mathcal{L}_{p}$ stability-in-expectation of the WNCS. For static protocols, we show that these conditions depend on the successful reception probabilities of the wireless channels. An advantage of our framework is that the obtained stability conditions can be directly used to study power control. We show that there are essential tradeoffs between the rate of transmission, transmitting power, and stability, and we illustrate how to allocate powers that can achieve stability for a given transmission rate. Lastly, we provide numerical examples to exhibit the virtue of our approach.

Our main contributions are threefold: 1) The proposed modelling framework is based on hybrid systems and it encompasses the linear models used in [6]-[9]. That is, we cover nonlinear plant and controller, stochastic and time-varying transmission instants, packet dropouts, intertransmission behaviour given by the continuous plant dynamics, and at-transmission behaviour given by packet transmissions. 2) We extend the nonlinear work in [12] to the case of multiple wireless channels each with a different successful reception probability. Our stability results are then significantly less conservative than the ones in [12] when the latter are applied to our setting. 3) We study transmitter power control, which to the best of our knowledge has not been done for general nonlinear systems in a closed-loop setting. 


\section{PRELIMINARIES AND PROBLEM DEFINITION}

\section{A. Notation}

Denote by $\mathbb{R}^{m \times n}$ the set of all real matrices of dimension $m \times n$. Let $\mathbb{R}_{\geq 0} \triangleq[0, \infty), \mathbb{R}_{>0} \triangleq(0, \infty), \mathbb{N} \triangleq\{0,1,2, \ldots\}$, and $\mathbb{N}_{>0} \triangleq \mathbb{N} \backslash\{0\}$. Given $t \in \mathbb{R}$ and a piecewise continuous function $f: \mathbb{R} \rightarrow \mathbb{R}^{n}$, we define $f\left(t^{+}\right) \triangleq \lim _{s \rightarrow t, s>t} f(s)$. For any $x \in \mathbb{R}^{n}$ and $y \in \mathbb{R}^{m}$, we use $(x, y) \triangleq\left[x^{\top} y^{\top}\right]^{\top} \in$ $\mathbb{R}^{n+m}$. For $x \in \mathbb{R}^{n},|x|$ denotes the standard Euclidean norm, and the same notation is used to denote the induced 2-norm of a matrix. The identity matrix of dimension $n \times n$ is denoted by $I_{n}$. The expectation and probability operators are denoted by $\mathbf{E}\{\cdot\}$ and $\operatorname{Pr}\{\cdot\}$, respectively. We write $x \sim \operatorname{Exp}(\alpha)$ to say that the random variable $x$ is exponentially distributed with parameter $\alpha$. The complementary error function is defined as $\operatorname{erfc}(x) \triangleq(2 / \sqrt{\pi}) \int_{x}^{\infty} \exp \left(-t^{2}\right) \mathrm{d} t$. Given a (Lebesgue) measurable function $f: \mathbb{R} \rightarrow \mathbb{R}^{n},\|f\|_{\mathcal{L}_{p}} \doteq$

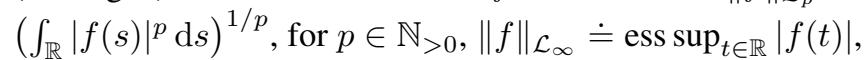

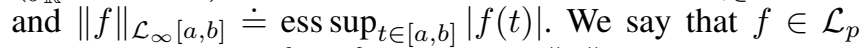
for $p \in \mathbb{N}_{>0} \cup\{+\infty\}$ whenever $\|f\|_{\mathcal{L}_{p}}<\infty$. Given $[a, b] \subset \mathbb{R},\|f\|_{\mathcal{L}_{p}[a, b]} \doteq\left(\int_{[a, b]}|f(s)|^{p} \mathrm{~d} s\right)^{1 / p}$ denotes the $\mathcal{L}_{p}$ norm of $f$ when restricted to the interval $[a, b]$.

\section{B. Stability notions}

We will consider hybrid systems of the form

$$
\begin{aligned}
\dot{z}(t) & =f(z(t), w(t)), t \in\left[t_{k}, t_{k+1}\right] \\
z\left(t_{k}^{+}\right) & =Q_{k}\left(z\left(t_{k}\right)\right), \\
y & =H(z),
\end{aligned}
$$

with state $z \in \mathbb{R}^{n_{z}}$, some prescribed output $y \in \mathbb{R}^{n_{y}}$, external disturbance $w \in \mathbb{R}^{n_{w}}$ which is assumed to belong to $\mathcal{L}_{p}$, a sequence of random maps $Q_{k}$, and $\left(t_{i+1}-t_{i}\right) \sim$ $\operatorname{Exp}(\boldsymbol{\omega})$ with parameter $\boldsymbol{\omega} \in \mathbb{R}_{>0}$. The definition of solutions to (1) can be found in [12], [23]. Similar hybrid systems have been used to model nonlinear stochastic WNCS, see e.g. [10]. For system (1), we introduce the following definition.

Definition 1: Let $p \in \mathbb{N}_{>0} \cup\{+\infty\}$ and $\gamma \geq 0$ be given. We say that (1) is $\mathcal{L}_{p}$ stable-in-expectation from $w$ to $y$ with gain $\gamma$ if there exists $K \geq 0$ such that $\mathbf{E}\left\{\|y\|_{\mathcal{L}_{p}\left[t_{0}, t\right]}\right\} \leq$ $K\left|z_{0}\right|+\gamma \mathbf{E}\left\{\|w\|_{\mathcal{L}_{p}\left[t_{0}, t\right]}\right\}$, for all $t \geq t_{0} \geq 0, z_{0} \in \mathbb{R}^{n_{z}}$, and $w \in \mathcal{L}_{p}$.

\section{Setup and problem definition}

Consider the nonlinear plant $\dot{x}_{p}=f_{p}\left(x_{p}, u, w\right), y=$ $g_{p}\left(x_{p}\right)$, where $x_{p} \in \mathbb{R}^{n_{p}}$ is the state of the plant, $u \in \mathbb{R}^{n_{u}}$ is the control input, $w \in \mathbb{R}^{n_{w}}$ is an external disturbance, $y \in \mathbb{R}^{n_{y}}$ is the plant output, and $\left(n_{p}, n_{u}, n_{w}, n_{y}\right) \in \mathbb{N}_{>0}^{4}$.

We follow an emulation approach [22], [23] and thus assume that a stabilising continuous-time nonlinear controller has been designed with model $\dot{x}_{c}=f_{c}\left(x_{c}, y, w\right), u=g_{c}\left(x_{c}\right)$, where $x_{c} \in \mathbb{R}^{n_{c}}$ is the controller state, and $n_{c} \in \mathbb{N}_{>0}$. The controller is then implemented over the wireless network as per the architecture in Fig. 1, for which it no longer receives $(y, u)$, but the networked versions $(\hat{y}, \hat{u})$, i.e. the vector of the most recently transmitted plant and controller output values.

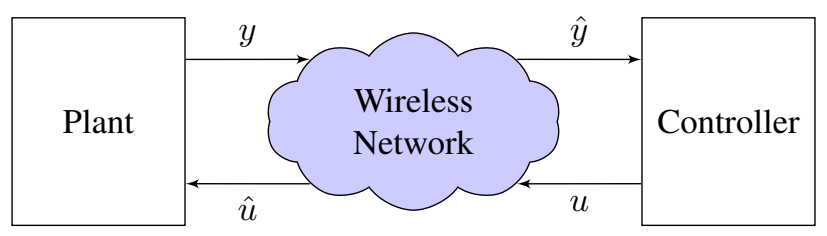

Fig. 1. Schematic of the wireless networked control system.

Therefore, with the wireless network, the plant and controller can be written as

$$
\begin{aligned}
\dot{x}_{p}=f_{p}\left(x_{p}, \hat{u}, w\right), \quad y=g_{p}\left(x_{p}\right), \\
\dot{x}_{c}=f_{c}\left(x_{c}, \hat{y}, w\right), \quad u=g_{c}\left(x_{c}\right) .
\end{aligned}
$$

The objectives of this work are to establish sufficient conditions in the network, in terms of data transmission rate, for which stability is preserved when the controller is implemented over the network, and to exploit these conditions to design suitable transmitter control policies.

\section{WIRELESS NETWORK MODEL}

The framework we present below can cover different types of wireless networks such as ad-hoc networks, which are essentially a collection of wireless nodes that can communicate at the same time [24]. The interference between devices can be very high and thus packet dropouts might have a significant impact on performance. Moreover, larger scale wireless networks such as $\mathrm{WiFi}$ or $5 \mathrm{G}$ small cells networks may also be subject to high interference.

\section{A. Transmission instants}

In wireless networks, because of synchronisation times, acknowledgements, etc., transmissions instants can be timevarying and random. Consequently, we assume the following.

Assumption 1: Consider a Poisson point process $r(t)$ with intensity $\boldsymbol{\omega}$ that satisfies $r(t)=0$ for $t \in\left[0, t_{0}\right)$ and $r(t)=k$ for $t \in\left[t_{k-1}, t_{k}\right)$. The sequence of transmission instants $\left\{t_{k}\right\}_{k \in \mathbb{N}}$ is defined inductively by: $t_{0}=\tau_{0}$ with $\tau_{0} \sim \operatorname{Exp}(\boldsymbol{\omega})$, and for each $k>0, t_{k}=t_{k-1}+\tau_{k}$, with $\tau_{k} \sim \operatorname{Exp}(\boldsymbol{\omega})$, where the sequence $\left\{\tau_{k}\right\}_{k \in \mathbb{N}}$ is i.i.d.

Poisson processes count the number of occurrences of some specific event through time. These are a natural modelling tool in numerous applied probability problems [25].

\section{B. Network-induced error}

An important object useful for our analysis is the so-called network-induced error, which is defined as $e \triangleq\left(e_{y}, e_{u}\right)$, where $e_{y} \triangleq \hat{y}-y$ and $e_{u} \triangleq \hat{u}-u$. We next define the concept of network node. A node consists of several sensors and/or actuators (grouped either by their spatial location or merely by convention) with their corresponding data being transmitted at the same transmission instant, we may also refer to this node as cluster. Consequently, we partition the network-induced error as $e=\left(e_{1}, \ldots, e_{N}\right)$ (after reordering, if needed), where $e_{n}, n \in\{1, \ldots, N\}$, is the network-induced error associated with the $n$-th node, and $N \in\left\{1, \ldots, n_{y}+n_{u}\right\}$ is the total number of nodes in the 
network. Each node may contain different links (we also refer to them as channels), thus we write $e_{n}=\left(e_{n, 1}, \ldots, e_{n, \ell_{n}}\right)$ (after re-ordering, if needed), for all $n=1, \ldots, N$ and associated partition $\ell_{n} \in\left\{1, \ldots, n_{y}+n_{u}\right\}$. We say that the WNCS has $N$ nodes and $\ell_{1}+\cdots+\ell_{N}$ channels.

\section{Interference model}

Consider that within a node $n$, every link $i_{n} \in\left\{1, \ldots, \ell_{n}\right\}$ has a transmitter and a receiver. Let the transmitter power of link $i_{n}$ be given by $p_{n, i_{n}} \in\left[0, P_{\max }\right], P_{\max } \in \mathbb{R}_{\geq 0}$ is the maximum allowable power at any given time instant, and the channel gain from link's $i_{n}$ transmitter to link's $j_{n}$ receiver be given by $g_{n, i_{n} j_{n}}$. Therefore, the signal-to-interferenceand-noise ratio (SINR) $\gamma_{n, i_{n}}$ perceived by the receiver of link $i_{n}$ (within node $n$ ) is given by (see e.g. [24])

$$
\gamma_{n, i_{n}} \triangleq \frac{g_{n, i_{n} i_{n}} p_{n, i_{n}}}{\sigma_{n, i_{n}}^{2}+\sum_{j_{n} \neq i_{n}} g_{n, j_{n} i_{n}} p_{n, j_{n}}},
$$

where $\sigma_{n, i_{n}}^{2}$ is the noise variance at the receiver of link $i_{n}$.

The probability of successful reception is a function the SINR, and we write $f_{n, i_{n}}$ in link $i_{n}$ and node $n$ as $f_{n, i_{n}}=$ $\psi\left(\gamma_{n, i_{n}}\right)$, where $\psi$ is a nonlinear function. In our examples we specify the model for both the probability and the channel gains, however they are not necessary for our results.

\section{Wireless network dynamics}

We now present the dynamics of the wireless network in terms of the network-induced error. Firstly, between transmission instants, we assume that $\hat{y}$ and $\hat{u}$ are generated by zero-order hold devices, i.e. $\dot{\hat{y}}=0$ and $\dot{\hat{u}}=0$ for $t \in\left[t_{k}, t_{k+1}\right]$ and $k \in \mathbb{N}$. Therefore, for $t \in\left[t_{k}, t_{k+1}\right]$,

$$
\dot{e}=\left[\begin{array}{c}
-\dot{y} \\
-\dot{u}
\end{array}\right]=\left[\begin{array}{l}
-\frac{\partial g_{p}}{\partial x_{p}} f_{p}\left(x_{p}, e_{u}+g_{c}\left(x_{c}\right), w\right) \\
-\frac{\partial g_{c}}{\partial x_{c}} f_{c}\left(x_{c}, e_{y}+g_{p}\left(x_{p}\right), w\right)
\end{array}\right] .
$$

Next we present the dynamics of $e$ at transmission instants. We assume that the network nodes are governed by a scheduling protocol. Consider that node $n$ is scheduled to transmit at time instant $t_{k}$. Then, we assume the corresponding error components associated with node $n, e_{n, i_{n}}$, $i_{n} \in\left\{1, \ldots, \ell_{n}\right\}$, will be set to zero only if the transmission is successful, i.e. $e_{n, i_{n}}\left(t_{k}^{+}\right)=0$, where $t_{k}^{+}$is the time instant immediately after the transmission (or "jump"). On the other hand, whenever a packet loss occurs, we assume the corresponding error components remain unchanged since the signal was not updated, i.e. $e_{n, i_{n}}\left(t_{k}^{+}\right)=e_{n, i_{n}}\left(t_{k}\right)$.

The above verbal description can be written as a mathematical equation for $e\left(t_{k}^{+}\right)$. We first introduce a random process that is useful to describe packet losses. Define $\Theta_{n}(k)=\operatorname{diag}\left\{\theta_{n, 1}(k), \ldots, \theta_{n, \ell_{n}}(k)\right\}$ for all $n=1, \ldots, N$, where each $\left\{\theta_{n, i_{n}}(k)\right\}_{k \in \mathbb{N}}$, is a sequence of independent random variables such that $\theta_{n, i_{n}}(k)=1$ with probability $f_{n, i_{n}}$, and $\theta_{n, i_{n}}(k)=0$ with probability $1-f_{n, i_{n}}$. Therefore, the dynamics of the network-induced error at jumps are

$$
\begin{aligned}
e\left(t_{k}^{+}\right)=\operatorname{diag} & \left\{\Theta_{1}(k), \ldots, \Theta_{N}(k)\right\} h\left(k, e\left(t_{k}\right)\right) \\
+ & \left(I-\operatorname{diag}\left\{\Theta_{1}(k), \ldots, \Theta_{N}(k)\right\}\right) e\left(t_{k}\right),
\end{aligned}
$$

where $h$ is a mapping that is determined by the implemented scheduling protocol. We refer to (5) as the protocol equation.

Assumption 2: Nodes are independent, i.e. $\left\{\Theta_{n}(k)\right\}_{k \in \mathbb{N}}$ and $\left\{\Theta_{m}(k)\right\}_{k \in \mathbb{N}}$ are independent for all $n \neq m$. Channels are independent at each node, i.e. $\left\{\theta_{n, i_{n}}(k)\right\}_{k \in \mathbb{N}}$ and $\left\{\theta_{n, j_{n}}(k)\right\}_{k \in \mathbb{N}}$ are independent for all $i_{n} \neq j_{n}$. We further assume that $\left\{\Theta_{n}(k)\right\}_{k \in \mathbb{N}}$ are independent of $\left\{\tau_{k}\right\}_{k \in \mathbb{N}}$.

Remark 1: We emphasise that our modelling framework covers a broader scenario than the one adopted in the previous work [12]. Particularly, (5) generalises the protocol equation found in [12], which is $e\left(t_{k}^{+}\right)=\theta(k) h\left(k, e\left(t_{k}\right)\right)+$ $(1-\theta(k)) e\left(t_{k}\right)$, where $\{\theta(k)\}_{k \in \mathbb{N}}$ is a scalar i.i.d. sequence of Bernoulli random variables such that $\theta(k)=1$ with probability $f$ and $\theta(k)=0$ with probability $1-f$. That is, in [12], the probability of success $f$ is assumed to be the same for every channel and every node, which is often unrealistic. On the contrary, we assume that every channel in the network has its own probability of successful transmission.

\section{Stability}

\section{A. Class of scheduling protocols}

In order to establish our stability result, we need the underlying scheduling protocols to be almost surely (a.s.) uniformly globally exponentially stable (UGES) as first introduced in [12], and we formalise it in the below definition.

Definition 2: Let $W: \mathbb{N} \times \mathbb{R}^{n_{e}} \rightarrow \mathbb{R}_{\geq 0}$ be given and suppose there exist a sequence of non-negative independent random variables $\left\{\kappa_{k}\right\}_{k \in \mathbb{N}}$, and numbers $a_{1}, a_{2}, \bar{\kappa} \in \mathbb{R}_{>0}$ such that the following conditions hold for the discrete-time system (5) for all $k \in \mathbb{N}$ and all $e \in \mathbb{R}^{n_{e}}$,

$$
\begin{aligned}
a_{1}|e| & \leq W(k, e) \leq a_{2}|e|, \\
W(k+1, h(k, e)) & \leq \kappa_{k} W(k, e), \\
\mathbf{E}\left\{\kappa_{k}\right\} & \leq \bar{\kappa}<1, \forall k \in \mathbb{N} .
\end{aligned}
$$

Then, we say that (5) is a.s. UGES with Lyapunov function $W$.

The definition above is the stochastic counterpart of the well-known UGES definition introduced in [23], where $\kappa_{k}=$ $\lambda$ for some $0 \leq \lambda<1$. Finding $\kappa_{k}$, and thus $\bar{\kappa}$, is done case by case, i.e. for a given scheduling protocol, we can find $\kappa_{k}$ and $\bar{\kappa}$ that satisfy the above definition.

Example 1 (Round-robin protocol): Nodes in the network are visited in a predetermined and cyclic manner [23]. For round-robin we have that $h$ in (5) is defined as $h(k, e) \triangleq$ $(I-\Delta(k)) e$, where $\Delta(k) \doteq \operatorname{diag}\left\{\delta_{1}(k) I_{s_{1}}, \ldots, \delta_{N}(k) I_{s_{N}}\right\}$, $\sum_{n=1}^{N} s_{n}=n_{e}$, and $\delta_{n}(k)=1$ if $k=n+\sigma N, \sigma \in$ $\mathbb{N}$, or $\delta_{n}(k)=0$ otherwise. Round-robin is UGES with $a_{1}=1, a_{2}=\sqrt{N}$, and $\lambda=\sqrt{(N-1) / N}$, see Proposition 4 in [23]. Note that $\kappa_{k} \in\{\lambda, 1\}$. Then, by definition of $\left\{\Theta_{n}(k)\right\}_{n=1, \ldots, N}$ we get that $\kappa_{k}$ is given by

$$
\begin{aligned}
\kappa_{k} \triangleq\left(\sum_{n=1}^{N}\left(\prod_{i_{n}=1}^{\ell_{n}} \theta_{n, i_{n}}(k)\right) \delta_{n}(k)\right) \lambda \\
\quad+1-\left(\sum_{n=1}^{N}\left(\prod_{i_{n}=1}^{\ell_{n}} \theta_{n, i_{n}}(k)\right) \delta_{n}(k)\right) .
\end{aligned}
$$

Given the definition of $\delta_{n}(k)$, only one element of the summation is active at transmission time $k$ (since only one 
node transmits per time instant). By using (7), we have that $\bar{\kappa}=1-\left(\min _{n \in\{1, \ldots, N\}} f_{n, 1} \cdots f_{n, \ell_{n}}\right)(1-\lambda)$.

\section{B. Main results}

We now show that under a.s. UGES protocols, $\mathcal{L}_{p}$ stabilityin-expectation of the WNCS in Fig. 1 is guaranteed with sufficiently frequent data transmission and some assumptions on (2). We first present the model for the overall WNCS in Fig. 1. Define the augmented state $x \triangleq\left(x_{p}, x_{c}\right)$, then by using (2), the definition of $e$, (4), and (5), we get

$$
\begin{aligned}
\dot{x}= & \mathbf{f}(x, e, w), t \in\left[t_{k}, t_{k+1}\right] \\
\dot{e}= & \mathbf{g}(x, e, w), t \in\left[t_{k}, t_{k+1}\right] \\
x\left(t_{k}^{+}\right)= & x\left(t_{k}\right), \\
e\left(t_{k}^{+}\right)= & \operatorname{diag}\left\{\Theta_{1}(k), \ldots, \Theta_{N}(k)\right\} h\left(k, e\left(t_{k}\right)\right) \\
& +\left(I-\operatorname{diag}\left\{\Theta_{1}(k), \ldots, \Theta_{N}(k)\right\}\right) e\left(t_{k}\right),
\end{aligned}
$$

where $\mathbf{g}$ is given by the right-side of (4), and $\mathbf{f}(x, e, w) \triangleq$ $\left(f_{p}\left(x_{p}, e_{u}+g_{c}\left(x_{c}\right), w\right), f_{c}\left(x_{c}, e_{y}+g_{p}\left(x_{p}\right), w\right)\right)$.

We will use the following proposition together with smallgain arguments to show $\mathcal{L}_{p}$ stability-in-expectation of the overall system (8).

Proposition 1: Consider the WNCS (8) and suppose the following holds.

(i) The protocol (8d) is a.s. UGES with Lyapunov function $W$ that is locally Lipschitz in $e$, uniformly in $k$.

(ii) There exists $L \geq 0$ such that

$$
\langle\partial W / \partial e, \mathbf{g}(x, e, w)\rangle \leq L W(k, e)+|\tilde{y}(x, w)|
$$

holds for almost all $e \in \mathbb{R}^{n_{e}}$, all $(x, w) \in \mathbb{R}^{n_{x}} \times \mathbb{R}^{n_{w}}$, $t \in\left(t_{k}, t_{k+1}\right)$, and $k \in \mathbb{N}$, where $\tilde{y}: \mathbb{R}^{n_{x}} \times \mathbb{R}^{n_{w}} \rightarrow$ $\mathbb{R}^{n_{e}}$ is a continuous function of $(x, w)$.

If the intensity of the intertransmission process $\boldsymbol{\omega}$ satisfies

$$
\boldsymbol{\omega}>L /(1-\bar{\kappa})
$$

then the error subsystem (8b)-(8d) is $\mathcal{L}_{p}$ stable-inexpectation from $\tilde{y}(x, w)$ to $W(e)$ with finite expected linear gain $\gamma_{e} \triangleq s_{\infty}(\boldsymbol{\omega}) /(\boldsymbol{\omega}-L)$, where $s_{\infty}(\boldsymbol{\omega}) \triangleq$ $\sum_{j=0}^{\infty}\left[\left(\frac{\omega}{\boldsymbol{\omega}-L}\right)^{j} \times \prod_{\iota=0}^{j-1} \mathbf{E}\left\{\kappa_{\iota}\right\}\right]<\infty$.

Note that (9) assumes an exponential growth on the $e-$ subsystem. It is satisfied when $W$ is Lipschitz in $e$ and $\mathbf{g}$ is globally Lipschitz for instance, see [23].

We can now state the main result of this paper. The proof follows immediately from Proposition 1 and the smallgain theorem for systems with jumps [23, Theorem 1]. The main idea behind the proof is to view system (8) as the interconnection of the $x$ - and $e$ - system through inputs $\tilde{y}(x, w)$ and $W(e)$.

Theorem 1: Consider the WNCS (8) and suppose the following holds.

( $i)$ The protocol (8d) is a.s. UGES with Lyapunov function $W$ that is locally Lipschitz in $e$, uniformly in $k$.

(ii) Condition $($ ii) of Proposition 1 holds with $\tilde{y} \triangleq G(x)+$ $E w$, for $G: \mathbb{R}^{n_{x}} \rightarrow \mathbb{R}^{n_{e}}$ and $E \in \mathbb{R}^{n_{e} \times n_{w}}$.

(iii) System (8a) is $\mathcal{L}_{p}$ stable from $(W(e), w)$ to $\tilde{y}(x, w)$ with finite gain $\gamma$ for some $p \in \mathbb{N}_{>0} \cup\{+\infty\}$.
If there exists an intensity of the inter-transmission process $\boldsymbol{\omega}$ that satisfies (10) and

$$
\gamma s_{\infty}(\boldsymbol{\omega}) /(\boldsymbol{\omega}-L)<1,
$$

then the WNCS (8) is $\mathcal{L}_{p}$ stable-in-expectation from $w$ to $(G(x), W(e))$ with finite linear gain.

Any stabilisable and detectable linear time-invariant system satisfies condition (iii). For examples of nonlinear systems that satisfy this condition we refer the reader to [26].

The rate of data transmission is measured in terms of the intensity $\boldsymbol{\omega}$ of the inter-transmission process. The required $\boldsymbol{\omega}$ that ensures $\mathcal{L}_{p}$ stability-in-expectation of system (8) can be easily computed numerically from Theorem 1 . We emphasise that the bound on $\boldsymbol{\omega}$ depends on the expectation $\mathbf{E}\left\{\kappa_{k}\right\}$. For static protocols, as shown in Section IV-A, we can find a closed form expression for $\mathbf{E}\left\{\kappa_{k}\right\}$ which depends on the cumulative probability of the network nodes.

We provide a closed-form bound on $\boldsymbol{\omega}$ in the corollary below, however, it is more conservative than (11).

Corollary 1: Suppose conditions $(i),($ ii $)$, and (iii) of Theorem 1 hold. If $\boldsymbol{\omega}$ satisfies

$$
\boldsymbol{\omega}>(\gamma+L) /(1-\bar{\kappa}),
$$

then the WNCS is $\mathcal{L}_{p}$ stable-in-expectation from $w$ to $(G(x), W(e))$ with finite linear gain.

The bound (12) is more conservative than (11) since it depends on $\bar{\kappa}$, which is the upper bound on $\mathbf{E}\left\{\kappa_{k}\right\}$. For instance, if we use a round-robin protocol, then $\bar{\kappa}$ depends on the smallest cumulative probability (i.e. worst overall channel quality) between the network nodes. In fact, the bounds in [12] are only applicable to our set-up if their probability of transmission is equal to the worst cumulative probability between the nodes, hence making [12] conservative when applied to a more general scenario.

\section{Power CONTROL IN WNCS}

Depending on the application, the transmission rate bounds provided in Section IV-B could be hard to satisfy, e.g. hardware not capable of handling it. However, these results can be used to tune the transmitter powers to ensure feasible transmission rates. A naive power control scheme would be using the maximum power in every transmitter, but this may not be viable since increasing power in turn increases the interference between channels, which may worsen the probability of success. In addition, maximum power causes serious energy consumption and electromagnetic pollution. We provide a more practical scheme which guarantees stability of the WNCS (8) for a given intensity of transmission.

To solve the power control problem, we use Corollary 1. From Assumption 1 we know that $\bar{\tau} \triangleq \mathbf{E}\left\{\tau_{k}\right\}=1 / \boldsymbol{\omega}$. That is, the reciprocal of the intensity of transmission can be thought of as the average maximum allowable transmission interval (MATI) that guarantees stability ${ }^{1}$. From Corollary 1 ,

\footnotetext{
${ }^{1}$ Note that our average MATI is different from the notion considered in the work [27], which is expressed in terms of a reverse average dwelltime condition for a deterministic setting. The value of our average MATI $\bar{\tau}$ depends on the particular application. For instance, $\bar{\tau}$ may represent a hardware constraint on how fast the network can physically transmit.
} 
and for a given $\bar{\tau}$, we have that any a.s. UGES protocol such that $\bar{\kappa}$ satisfies $0<\bar{\kappa}<1-\bar{\tau}(\gamma+L)$ ensures $\mathcal{L}_{p}$ stability-in-expectation of (8). Now, suppose we use a round-robin protocol (or any other periodic static protocol) to schedule transmissions, then by Example 1 we have that $\bar{\kappa}$ depends on the minimum cumulative probability between the nodes. That is, it depends on the node with the worst overall channel quality, which we denote by $\mathbf{n}$. Then the required probabilities that ensure stability for a given $\bar{\tau}$ satisfy

$$
f_{\mathbf{n}, 1} \cdots f_{\mathbf{n}, \ell_{\mathbf{n}}}>\frac{\bar{\tau}(\gamma+L)}{1-\lambda} .
$$

In wireless communications, the objective of power control is to adjust the transmitter powers of all users such that the SINR of each user meets a given threshold required for acceptable performance [24]. Note that (13) is essentially a threshold on SINR since success probabilities are related to SINR. In fact, we can state the following lemma.

Lemma 1: If there exists a set of transmitter powers $\left\{p_{\mathbf{n}, 1}, \ldots, p_{\mathbf{n}, \ell_{\mathbf{n}}}\right\}$ such that

$$
\prod_{i_{\mathbf{n}}=1}^{\ell_{\mathbf{n}}} \psi\left(\frac{g_{\mathbf{n}, i_{\mathbf{n}} i_{\mathbf{n}}} p_{\mathbf{n}, i_{\mathbf{n}}}}{\sigma_{\mathbf{n}, i_{\mathbf{n}}}^{2}+\sum_{j_{\mathbf{n}} \neq i_{\mathbf{n}}} g_{\mathbf{n}, j_{\mathbf{n}} i_{\mathbf{n}}} p_{\mathbf{n}, j_{\mathbf{n}}}}\right)>\frac{\bar{\tau}(\gamma+L)}{1-\lambda},
$$

and $0 \leq p_{\mathbf{n}, i_{\mathbf{n}}} \leq P_{\max }, i_{\mathbf{n}}=1, \ldots, \ell_{\mathbf{n}}$. Then, (8) is $\mathcal{L}_{p}$ stable-in-expectation transmitting data every $\bar{\tau}$ seconds in average.

Lemma 1 provides a practical way of finding transmitter powers to achieve stability for a given $\bar{\tau}$. We show in the next section that this can be easily done numerically for given models of success probability $\psi$ and channel gains $g_{\mathbf{n}, i_{\mathbf{n}} i_{\mathbf{n}}}$. Moreover, we could formulate an optimisation problem which objective is to use minimal transmission power to achieve stability. That is, find $\left\{p_{\mathbf{n}, 1}, \ldots, p_{\mathbf{n}, \ell_{\mathbf{n}}}\right\}$ that minimise some cost function $J\left(p_{\mathbf{n}, 1}, \ldots, p_{\mathbf{n}, \ell_{\mathbf{n}}}\right)$ under constraints (14) and $0 \leq p_{\mathbf{n}, i_{\mathbf{n}}} \leq P_{\max }, i_{\mathbf{n}}=1, \ldots, \ell_{\mathbf{n}}$. However, this is out of the scope of this paper.

\section{NUMERICAL EXAMPLES}

We illustrate our results in the control of an unstable batch reactor over wireless channels. This is a two-input two-output system, and its model parameters can be found in [28, p.62]. Particularly, the plant is given by $\dot{x}_{p}=A_{p} x_{p}+B_{p} u, y=$ $C_{p} x_{p}$, which is stabilised by a PI controller with the statespace representation $\dot{x}_{c}=A_{c} x_{c}+B_{c} y, u=C_{c} x_{c}+D_{c} y$. The wireless network is composed of two nodes $N=2$, and each node has two channels $\ell_{1}=\ell_{2}=2$. We adopt the round-robin protocol in Example 1. We send the two output measurements through the two channels in Node 1, and the two control inputs through the two channels in Node 2. Therefore, $e=\left(e_{1}, e_{2}\right)$, where $e_{1}=\left(\hat{y}_{1}-y_{1}, \hat{y}_{2}-y_{2}\right)$ and $e_{2}=\left(\hat{u}_{1}-u_{1}, \hat{u}_{2}-u_{2}\right)$. We can thus write the dynamics

$$
\begin{aligned}
& \dot{x}=A_{11} x+A_{12} e, \\
& \dot{e}=A_{21} x+A_{22} e,
\end{aligned}
$$

$A_{21} \triangleq-\operatorname{diag}\left\{C_{p}, C_{c}\right\} A_{11}, A_{22} \triangleq-\operatorname{diag}\left\{C_{p}, C_{c}\right\} A_{12}$, $A_{11} \triangleq\left[\begin{array}{cc}A_{p}+B_{p} D_{c} C_{p} B_{p} C_{c} \\ B_{c} C_{p} & A_{c}\end{array}\right], A_{12} \triangleq\left[\begin{array}{cc}B_{p} D_{c} B_{p} \\ B_{c} & 0\end{array}\right]$. We now find the parameters required to compute the bounds in Theorem 1 and Corollary 1. From Example 1, we have that $\lambda=\sqrt{0.5}$. Similar to Example 3 and Section IX in [23] we get $L=\sqrt{N}\left|A_{22}\right|=25.27$ and $\gamma=27.34$.

\section{A. Comparison between the transmission bounds}

Suppose the wireless network is initially configured such that the probabilities of sucessful transmission are given by $f_{1,1}=0.3, f_{1,2}=0.8, f_{2,1}=0.75$, and $f_{2,2}=0.8$. From Example 1 we get $\bar{\kappa}=1-(0.3 \times 0.8)(1-\sqrt{0.5})=0.93$, and $\mathbf{E}\left\{\kappa_{k}\right\}=0.93$ for $k=1+2 \sigma$, and $\mathbf{E}\left\{\kappa_{k}\right\}=0.82$ for $k=2+$ $2 \sigma, \sigma \in \mathbb{N}$. We compute the numerical bound from Theorem 1 , which can be derived by solving numerically for $\boldsymbol{\omega}$ in (11), giving a value of $\boldsymbol{\omega}_{\text {num }}^{*}=428.64$. Then, we compute the closed-form stability bound from Corollary 1 using (12), giving us $\boldsymbol{\omega}_{\text {closed }}^{*}=748.45$. We can see that $\boldsymbol{\omega}_{\text {closed }}^{*} / \boldsymbol{\omega}_{\text {num }}^{*}=1.75$, i.e. the closed-form bound is around twice more conservative than the numerical bound. Recall from the discussion at the end of Section IV-B, that $\boldsymbol{\omega}_{\text {closed }}^{*}$ also corresponds to the bound in [12] if we were to use it in our setting. That is, the bound in [12] is twice more conservative when applied to more general scenarios, which highlights our results.

These intensity values can be translated into average MATI bounds since $\mathbf{E}\left\{\tau^{*}\right\}=1 / \boldsymbol{\omega}^{*}$. The closed-form bound requires the network to transmit packets, on average, every $1.3[\mathrm{~ms}]$, and every $2.3[\mathrm{~ms}]$ for the less conservative bound.

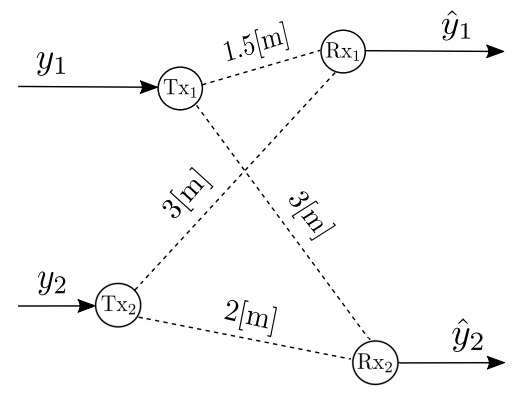

Fig. 2. Physical placement of the transmitters and receivers of channels one and two at Node 1.

\section{B. Power control for a given average MATI}

To model probability of success we choose $\psi\left(\gamma_{n, i_{n}}\right) \triangleq$ $\left(1-0.5 \operatorname{erfc}\left(\gamma_{n, i_{n}}\right)\right)^{\mathrm{M}}$, where $\mathrm{M}$ is the packet size in bits and $\operatorname{erfc}(\cdot)$ is the complementary error function. Suppose that Node 1 has the worst (lowest) cumulative probability, that its transmitters and receivers are physically placed as per Fig. 2, and that we require an average MATI of $\bar{\tau}=1[\mathrm{~ms}]$. Then, by Lemma 1 , we need to find $p_{1,1}$ and $p_{1,2}$ such that

$$
\begin{aligned}
& \left(1-0.5 \operatorname{erfc}\left(\frac{g_{1,11} p_{1,1}}{\sigma^{2}+g_{1,21} p_{1,2}}\right)\right)^{\mathrm{M}} \\
& \times\left(1-0.5 \operatorname{erfc}\left(\frac{g_{1,22} p_{1,2}}{\sigma^{2}+g_{1,12} p_{1,1}}\right)\right)^{\mathrm{M}}>0.1057 .
\end{aligned}
$$

We compute the channel gains by using $g_{1, i j}=1 / d_{1, i j}^{4}$ as in [29], where $d_{1, i j}$ is the distance between the transmitter in channel $i \in\{1,2\}$ and the receiver in channel $j \in\{1,2\}$ (both within Node 1). Given Fig. 2, we get 
$g_{1,11}=0.2, g_{1,22}=0.063$, and $g_{1,12}=g_{1,21}=0.012$. We pick $\sigma^{2}=1, \mathrm{M}=1024$ bits, and $P_{\max }=70$. We plot the left-hand side of (16) for different values of powers $p_{1,1}, p_{1,2}$ as illustrated in Fig. 3 . We have also plotted the plane $f_{1,1} \times f_{1,2}=0.1057$ to illustrate the stabilising region, which is the region above such plane.

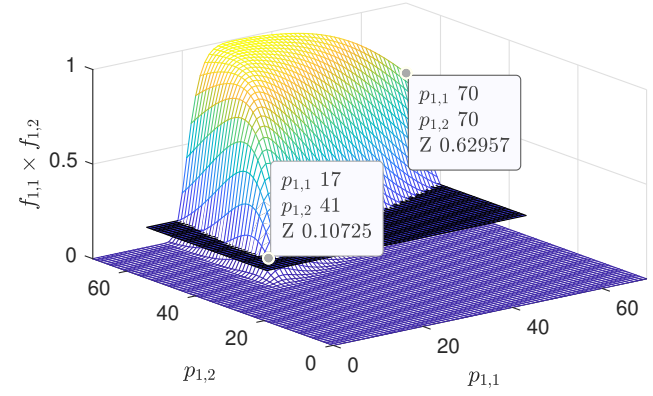

Fig. 3. Probabilities of successful transmission with respect to transmitter powers at Node 1 .

Note from the data-tips in Fig. 3 that the naive strategy of setting $p_{1,1}=p_{1,2}=P_{\max }$ ensures stability as one would expect provided there is enough available power. However, our results illustrate that a more advanced strategy can guarantee stability with significantly less power. For instance, setting $p_{1,1}=17$ and $p_{1,2}=41$ ensures stability with about $76 \%$ less power usage at transmitter $\mathrm{Tx}_{1}$, and $41 \%$ at $\mathrm{Tx}_{2}$.

\section{CONCLUSIONS}

We provided sufficient conditions on the intensity of transmission that guarantee $\mathcal{L}_{p}$ stability-in-expectation of a nonlinear WNCS subject to multiple packet losses. These are control-oriented results but they try to bridge the gap between control and communication literature by carefully modelling the network and connecting the main result to power control via realistic and well-known interference models. In our approach, the stability and power control problems are coupled a posteriori, and future work involves studying the co-design problem of stability and power control for our current setting.

\section{REFERENCES}

[1] P. Park, S. C. Ergen, C. Fischione, C. Lu, and K. H. Johansson, "Wireless network design for control systems: A survey," IEEE Communications Surveys \& Tutorials, vol. 20, no. 2, pp. 978-1013, 2017.

[2] L. Schenato, B. Sinopoli, M. Franceschetti, K. Poolla, and S. Sastry, "Foundations of control and estimation over lossy networks," Proceedings of the IEEE, vol. 95, no. 1, pp. 163 - 187, January 2007.

[3] M. Moayedi, Y. K. Foo, and Y. C. Soh, "Networked LQG control over unreliable channels," International Journal of Robust and Nonlinear Control, vol. 23, no. 2, pp. 167-189, 2013.

[4] J. Moon and T. Başar, "Minimax control over unreliable communication channels," Automatica, vol. 59, pp. 182-193, 2015.

[5] H. Lin, H. Su, P. Shi, Z. Shu, R. Lu, and Z.-G. Wu, "Optimal estimation and control for lossy network: stability, convergence, and performance," IEEE Transactions on Automatic Control, vol. 62, no. 9, pp. 4564-4579, 2017.

[6] E. Garone, B. Sinopoli, A. Goldsmith, and A. Casavola, "LQG control for MIMO systems over multiple erasure channels with perfect acknowledgment," IEEE Transactions on Automatic Control, vol. 57, no. 2, pp. 450-456, 2012.
[7] W. Chen and L. Qiu, "Linear quadratic optimal control of continuoustime lti systems with random input gains," IEEE Transactions on Automatic Control, vol. 61, no. 7, pp. 2008-2013, 2015.

[8] M. Rich and N. Elia, "Optimal mean-square performance for mimo networked systems," in 2015 American Control Conference (ACC). IEEE, 2015, pp. 6040-6045.

[9] A. Maass, F. Vargas, and E. Silva, "Optimal control over multiple erasure channels using a data dropout compensation scheme," Automatica, vol. 68, pp. 155-161, 2016.

[10] J. P. Hespanha and A. R. Teel, "Stochastic impulsive systems driven by renewal processes," in 17th International Symposium on Mathematical Theory of Networks and Systems (MTNSO6), 2006.

[11] D. E. Quevedo and D. Nešić, "Robust stability of packetized predictive control of nonlinear systems with disturbances and markovian packet losses," Automatica, vol. 48, no. 8, pp. 1803-1811, 2012.

[12] M. Tabbara and D. Nešić, "Input-output stability of networked control systems with stochastic protocols and channels," IEEE Transactions on Automatic control, vol. 53, no. 5, pp. 1160-1175, 2008.

[13] C. Saraydar, N. Mandayam, D. Goodman et al., "Pricing and power control in a multicell wireless data network," IEEE Journal on selected areas in communications, vol. 19, no. 10, pp. 1883-1892, 2001.

[14] S. Kandukuri and S. Boyd, "Optimal power control in interferencelimited fading wireless channels with outage-probability specifications," IEEE transactions on wireless communications, vol. 1, no. 1, pp. 46-55, 2002.

[15] C. Zhang, A. Agrawal, V. S. Varma, and S. Lasaulce, "Thresholdingbased distributed power control for energy-efficient interference networks," in 2018 IEEE 29th Annual International Symposium on Personal, Indoor and Mobile Radio Communications (PIMRC). IEEE, 2018, pp. 1-6.

[16] D. Quevedo, A. Ahlén, A. Leong, and S. Dey, "On kalman filtering over fading wireless channels with controlled transmission powers,' Automatica, vol. 48, no. 7, pp. 1306-1316, 2012.

[17] X. Ren, J. Wu, K. H. Johansson, G. Shi, and L. Shi, "Infinite horizon optimal transmission power control for remote state estimation over fading channels," IEEE Transactions on Automatic Control, vol. 63 , no. 1, pp. 85-100, 2017.

[18] Y. Li, J. Wu, and T. Chen, "Transmit power control and remote state estimation with sensor networks: A bayesian inference approach," Automatica, vol. 97, pp. 292-300, 2018

[19] K. Gatsis, A. Ribeiro, and G. J. Pappas, "Optimal power management in wireless control systems," IEEE Transactions on Automatic Control, vol. 59, no. 6, pp. 1495-1510, 2014.

[20] V. S. Varma, A. M. de Oliveira, R. Postoyan, I.-C. Morarescu, and J. Daafouz, "Energy-efficient time-triggered communication policies for wireless networked control systems," IEEE Transactions on Automatic Control, 2019.

[21] K. Gatsis, A. Ribeiro, and G. J. Pappas, "Random access design for wireless control systems," Automatica, vol. 91, pp. 1-9, 2018.

[22] G. Walsh, O. Beldiman, and L. Bushnell, "Asymptotic behavior of nonlinear networked control systems," IEEE Transactions on Automatic Control, vol. 46, no. 7, pp. 1093-1097, jul 2001.

[23] D. Nešić and A. Teel, "Input-output stability properties of networked control systems," IEEE Transactions on Automatic Control, vol. 49, no. 10, pp. 1650-1667, 2004.

[24] A. Goldsmith, Wireless Communications. Cambridge University Press, 2005

[25] H. C. Tijms, A first course in stochastic models. John Wiley and sons, 2003.

[26] M. Abdelrahim, R. Postoyan, J. Daafouz, and D. Nešić, "Stabilization of nonlinear systems using event-triggered output feedback controllers," IEEE Transactions on Automatic Control, vol. 61, no. 9, pp. 2682-2687, 2016.

[27] S. H. Heijmans, R. Postoyan, D. Nešić, and W. Heemels, "An average allowable transmission interval condition for the stability of networked control systems," IEEE Transactions on Automatic Control, 2020.

[28] M. Green and D. J. Limebeer, Linear robust control. Courier Corporation, 2012

[29] T. ElBatt and A. Ephremides, "Joint scheduling and power control for wireless ad hoc networks," IEEE Transactions on Wireless communications, vol. 3, no. 1, pp. 74-85, 2004. 